\title{
The efficacy of preoperative PET/CT for prediction of curability in surgery for locally advanced gastric carcinoma
}

\author{
Hoon Hur', Sung Hoon Kim², Wook Kim³ ${ }^{3}$ Kyo Young Song ${ }^{3}$, Cho Hyun Park ${ }^{3}$, Hae Myung Jeon ${ }^{3 *}$
}

\begin{abstract}
Background: The benefits of preoperative ${ }^{18} \mathrm{FDG}-\mathrm{PET} / \mathrm{CT}$ for gastric cancer remain uncertain. The aim of this study was to investigate the effects of preoperative ${ }^{18} \mathrm{FDG}-\mathrm{PET} / \mathrm{CT}$ on the surgical strategy for locally advanced gastric cancer retrospectively.

Methods: From January 2007 to November 2008, ${ }^{18}$ FDG-PET/CT was performed in 142 patients who had been diagnosed with advanced gastric cancer by computed tomography or gastrofiberscope findings.

Results: Detection rates were 88.7\% (126/142) for primary tumors and 24.6\% (35/142) for local lymph nodes (LN). Nine patients with metastatic lesions underwent induction chemotherapy without operation. Of 133 patients subjected to operation, positive FDG uptake in primary tumors $(p=0.047)$ and local lymph nodes $(p<0.001)$ was related to non-curable operations. The mean standard uptake value (SUV) of primary tumors of patients who underwent non-curable operations was significantly higher than that of patients with curable operations ( $p=0.001$ ). When the SUV was greater than 5 and FDG uptake of LN was positive, non-curable operations were predicted with a sensitivity of $35.2 \%$, a specificity of $91.0 \%$ and an accuracy of $76.7 \%$.

Conclusions: High SUV of the primary tumor and positive FDG uptake in local lymph nodes at PET/CT could predict non-curative resection in locally advanced gastric cancer. Therefore, information from preoperative PET/CT can help physician decisions regarding other modalities without laparotomy.
\end{abstract}

\section{Background}

Preoperative imaging studies are used to evaluate clinical and surgical factors of malignant tumors, including resectability and identification of metastatic lesions that contraindicate resection. Although the presence of locoregional disease in imaging studies will direct the surgical oncologist toward exploration with the intention of complete resection, the ability of these studies to exclude non-curability in surgery remains controversial.

In gastric cancer, the primary aim of surgery is curability, i.e., elimination of macroscopic and microscopic remnants of the malignant tumor by resection of the stomach and proper lymphadenectomy [1]. Since noncurative treatment is a definite poor prognostic factor for patients who undergo surgery for gastric cancer $[2,3]$,

\footnotetext{
* Correspondence: hmjeon@catholic.ac.kr

${ }^{3}$ Department of Surgery, The Catholic University of Korea, College of

Medicine, Seoul, Korea

Full list of author information is available at the end of the article
}

other modalities may be needed in order to increase their survival. However, it is not easy to preoperatively diagnose non-curability by conventional non-invasive imaging methods such as computed tomography (CT), endoscopic ultrasound (EUS) and magnetic resonance imaging (MRI) without laparotomy or laparoscopic staging under general anesthesia.

Positron emission tomography (PET) imaging using the radiolabeled glucose analog ${ }^{18}$ fluorodeoxyglucose (FDG) can present biologic images according to glucose metabolism. PET imaging can be combined with anatomic imaging such as conventional CT scanning in order to increase diagnostic accuracy [4]. Although the National Comprehensive Cancer Network (NCCN) recently announced that preoperative PET/CT for gastric cancer patients can be recommended as an option of preoperative staging [5], the benefits of PET/CT remain uncertain.

Therefore, we analyzed information from preoperative PET/CT for patients with locally advanced gastric 
cancer and compared it with the surgical results, retrospectively. Uptake of FDG in the primary tumor or local lymph node and the standardized uptake value (SUV) were investigated for their potential in preoperative prediction of non-curative surgery. Thus, the aim of this study was to investigate the effects of preoperative PET/ $\mathrm{CT}$ on the surgical strategy in gastric cancer patients.

\section{Methods}

\section{Patient selection and study}

From January 2007 to November 2008, our institution performed whole body ${ }^{18} \mathrm{~F}$-FDG PET/CT scans for 142 consecutive patients about three days before surgery. These patients had been pathologically diagnosed with gastric adenocarcinoma by endoscopic biopsy and suspected of having advanced gastric cancer by endoscopic findings or conventional enhanced CT scans. They underwent careful physical examinations and other imaging studies such as bone scans and chest radiography in order to exclude distant metastasis. We obtained written informed consent from the patients for preoperative $\mathrm{PET} / \mathrm{CT}$, and then collected their preoperative staging data and surgical results for this retrospective study.

\section{$\mathrm{PET} / \mathrm{CT}$ imaging}

Before PET/CT scanning, all patients fasted for at least 6 hours. Patients were confirmed to have blood sugar levels below $130 \mathrm{mg} / \mathrm{mL}$ and rested for approximately 45 minutes before receiving an intravenous injection of $440 \mathrm{MBq}$ of 18F-FDG. Scanning began 60 minutes later. A combined PET/CT in-line system (Biograph LSD, Siemens, Knoxville, TN) was used for all data collection. CT scanning was performed from the orbitomeatal line to the upper thigh $(30 \mathrm{~mA} ; 130 \mathrm{kV} ; 5 \mathrm{~mm}$-thick sections) prior to PET. PET was then immediately conducted over the same body region with 6-8 bed positions, with 2 min acquisition time per bed position.

\section{Interpretation of PET/CT}

$\mathrm{PET} / \mathrm{CT}$ images were reviewed at a workstation with fusion software (Syngo, Siemens, Knoxville, TN) by a nuclear medicine physician who was given information about the clinical findings in the patient. The images were analyzed for the site and amount of positive FDG uptake; FDG uptake was defined as qualitatively positive when focal uptake was higher than normal background FDG activity in the primary tumor, local lymph node and metastatic lesions. FDG uptake in the bowel was regarded as positive when there was wall thickening of the same bowel at CT scan. The FDG uptake activity within each lesion was corrected by the administered dose and the patient weight to produce a maximum standardized uptake value (SUV). For this study, we only evaluated the SUV to primary tumors.

\section{Conventional CT scan}

Conventional abdominal enhanced CT scanning (LightSpeed VCT, GE Healthcare, Milwaukee, WI) was performed after intravenous administration of contrast agents, with 5- to 10 - $\mathrm{mm}$ slice thickness from the diaphragm to the symphysis pubis. The image was also reviewed by a radiologist who was provided with patient information. Non-curable operation was defined on CT scans when suspicious findings met the criterion of metastatic or non-resectable primary tumors in the surgical strategy.

\section{Treatment Plan}

In our institution, we have the following treatment strategy for gastric cancer: patients who have metastatic lesions in either PET/CT or CT are started on induction chemotherapy with or without pathologic confirmation. Metastatic lesions of gastric cancer include liver and retroperitoneal lymph nodes or seeding into the peritoneum. A non-resectable primary tumor is indicated by pancreatic or duodenal invasion requiring pancreaticoduodenectomy, or invasion into the root of the mesocolon. Cases with only one modality of PET/CT and CT showing metastatic or non-resectable primary tumors undergo additional imaging studies such as magnetic resonance image (MRI) and ultrasound (US). Patients with suspicious metastatic lesions in the imaging study are subjected to surgical staging.

\section{Surgery}

If the patient had suspicious metastatic lesions or a nonresectable primary tumor in the imaging studies, we first performed a minilaparotomy in order to confirm metastasis or the possibility of resectability. The abdominal incision was extended in cases with resectability in the surgical findings, and then surgery was performed by conventional open gastrectomy with over D1 plus beta lymphadenectomy with the intention of curability. Noncurable operation was defined when we performed open and close bypass surgery without tumor resection due to metastatic lesions in other organs, the peritoneum and retroperitoneal lymph, or when non-resectable primary tumors were found during surgery. In addition, palliative resection of primary tumors in which microscopic (R1) or macroscopic (R2) tumors remained was also included in the category of non-curative operation.

\section{Statistical analysis}

Statistical analysis was performed with the statistical package for social sciences (SPSS) version 13.0. A Chi-square test was performed in order to evaluate differences of FDG uptake rates in primary tumors or local lymph nodes according to the clinico-pathological factors. The SUVs of curable and non-curable operations were compared by an 
independent $\mathrm{t}$-test. The extent to which the SUV differed between a curable and non-curable operation was assessed using receiver operator characteristics (ROC) plots. We plotted ROC curves for SUV to predict non-curable operation, and then calculated sensitivity, specificity, accuracy and the positive predictive value at different SUV cutoffs (5, 7 and 9) as well as positive uptake of local lymph nodes.

\section{Results}

In 142 enrolled patients, the FDG uptake rate of primary tumors was $88.7 \%(126 / 142)$ and that of local lymph nodes was $24.6 \%(32 / 142)$. The mean SUV of primary cancers was 5.7 (range, 1.89-19.06). In 2 patients, other simultaneous malignancies (thyroid cancer and rectal cancer) that the other imaging study could not detect were incidentally found. We performed combined operations for those patients.

Nine patients who had metastatic lesions or nonresectable primary tumors in either PET/CT or conventional CT scan were not operated on. The PET/CT findings of these patients are listed in Table 1, showing that all patients had positive FDG uptake in the primary tumor. We performed operations on the remaining 133 patients and then evaluated the possibility of curative surgery (Fig 1).

The clinico-pathological characteristics of the 133 patients who underwent surgery are presented in Table 2. The rates of FDG uptake in the primary tumor and local lymph nodes were compared according to age, gender, diabetic mellitus, tumor size, tumor location, histology and curability of operations. Except for noncurative operation $(97.1 \%$ vs. $84.8 \%, p=0.047)$, no factors were significantly correlated with the FDG uptake rate in the primary tumor. Patients with large tumor sizes showed relatively high uptake rates in the primary tumor $(92.6 \%$ vs. $83.1 \%, p=0.090)$. The FDG uptake rate of local lymph nodes was significantly higher in patients who underwent non-curative operations (44.1\% vs. $14.1 \%, p<0.001$ ).

The mean maximum SUV of primary tumors in patients with non-curative operations was $7.3 \pm 4.5$ (mean \pm S.D.) and that of patients with curative operations was $4.4 \pm 3.5$ (mean \pm S.D.). The difference in SUV between the two groups was significant $(\mathrm{p}=$ 0.001 ), and a box plot of the SUVs in both groups is presented in Fig 2A.

An ROC curve of the maximum SUV was plotted in order to predict non-curative operations, and an area under the curve of 0.730 ( $p<0.001 ; 0.629<95 \%$ C.I. $<$ 0.831) was obtained (Fig 2B). We calculated diagnostic indices (sensitivity, specificity, accuracy and positive predictive value) at various SUV cutoffs for primary tumor and lymph node FDG uptake, and then compared these results with predictions from conventional enhanced CT scanning. When the maximum SUV was greater than 5 and the FDG uptake of lymph node was positive, noncurative operation was predicted with a sensitivity of $35.2 \%$, a specificity of $91.0 \%$, an accuracy of $76.7 \%$ and a positive predictive value of $57.1 \%$. These values are higher than those obtained using other SUV cutoffs for primary tumors or even with conventional enhanced CT scanning (sensitivity of $17.6 \%$, specificity of $87.9 \%$, accuracy of $69.9 \%$ and a positive predictive value of $33.3 \%$ ) (Table 3).

\section{Discussion}

For patients with locally advanced gastric cancer, the preoperative prediction of curability is important because it can prevent unnecessary laparotomies and

Table 1 Study results of patients who underwent induction chemotherapy without operation

\begin{tabular}{|c|c|c|c|c|c|}
\hline \multirow[t]{2}{*}{ No } & \multirow[t]{2}{*}{$C T$ finding } & \multicolumn{3}{|c|}{ PET/CT finding } & \multirow[t]{2}{*}{ Additional study } \\
\hline & & $\begin{array}{l}\text { Primary } \\
\text { SUV }\end{array}$ & $\begin{array}{l}\text { Local LN } \\
\text { SUV }\end{array}$ & Other uptake & \\
\hline 1 & Lung metastasis & 2.97 & 2.97 & Lung, Bone & Spine MRI \\
\hline 2 & $\begin{array}{l}\text { Peritoneal seeding } \\
\text { Liver metastasis }\end{array}$ & 6.81 & 5.44 & Mesentery & \\
\hline 3 & $\begin{array}{l}\text { Peritoneal seeding } \\
\text { Esophagus invasion }\end{array}$ & 7.89 & 0 & Distal Esophagus & \\
\hline 4 & Peritoneal seeding & 3.91 & 3.34 & Peritoneum & \\
\hline 5 & Peritoneal seeding & 3.73 & 8.15 & $\begin{array}{l}\text { Retroperitoneal LN } \\
\text { Lt. supraclaviclar LN }\end{array}$ & \\
\hline 6 & Liver metastasis & 10.73 & 0 & Liver & Sono, Liver MRI \\
\hline 7 & Liver metastasis & 7.26 & 12.18 & Liver & Sono \\
\hline 8 & Peritoneal seeding & 2.4 & 0 & $\begin{array}{l}\text { T-colon, Omentum, } \\
\text { Retroperitoneal LN }\end{array}$ & \\
\hline 9 & Liver metastasis & 11.9 & 0 & Liver & \\
\hline
\end{tabular}

PET, positron emission tomography, CT, computed tomography, LN, lymph nodes, SUV, standardized uptake value, MRI, magnetic resonance imaging. 


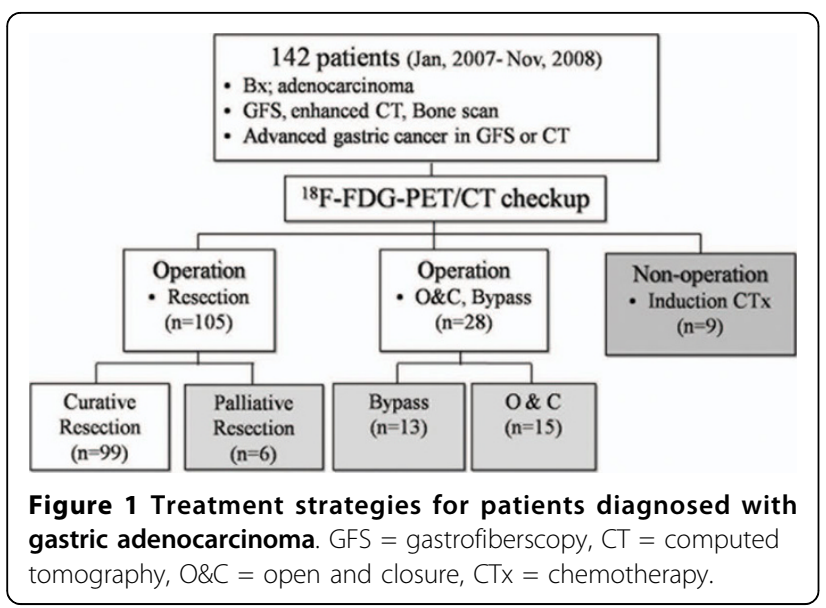

direct physicians toward treatment with other modalities such as neoadjuvant chemotherapy. Conventional enhanced CT scans are one of the most important imaging methods for preoperative prediction of curability. Therefore, patients diagnosed with definite metastatic lesions (cM1) by CT scan might be treated systemically without surgery. However, the treatment strategy for patients with locally advanced gastric cancer and without definite cM1 lesions has often been decided based on surgical findings following laparotomy or laparoscopic staging [6]. Our results in patients with locally advanced gastric cancer show that preoperative ${ }^{18}$ F-FDG PET/CT could provide objective information for decisions regarding treatment strategies such as laparoscopic staging and neoadjuvant chemotherapy.

At present, several studies have reported that FDGPET is the most sensitive non-invasive imaging strategy for detecting distant metastasis $[7,8]$. Therefore, our study was also designed that patients with suspected metastatic lesions on CT scanning accompanied by FDG uptake were started on induction chemotherapy without operation. Previous studies reported that FDG-PET, and not PET/CT, was more sensitive than CT scanning for detecting primary tumors in advanced disease, but inferior to CT for detecting intra-abdominal lymph node metastasis $[8,9]$. In addition, recent studies showed that FDG-PET had lower sensitivity for detection of lymph nodes metastasis, and even had no definite role as preoperative imaging in gastric cancer $[10,11]$. Moreover, studies validating the use of PET/CT in gastric carcinoma are lacking thus far, and most physicians cannot confirm whether adding CT information to FDG-PET will improve diagnostic accuracy. Due to these reasons, the current aims of preoperative PET/CT in most centers that perform operations for gastric cancer patients, including our institution, are as follows: 1) to confirm metastasis by contrast-enhanced CT scan; 2) to investigate metastatic lesions that are not detected by contrast-

Table 2 Preoperative and operative findings of PET/CT in patients who underwent operation $(\mathbf{n}=133)$

\begin{tabular}{|c|c|c|c|c|c|c|c|c|}
\hline & & \multirow[t]{2}{*}{$n$} & \multicolumn{3}{|c|}{ FDG uptake in primary tumor } & \multicolumn{3}{|c|}{ FDG uptake in local LN } \\
\hline & & & $\begin{array}{c}\text { Yes (\%) } \\
(n=117)\end{array}$ & $\begin{array}{c}\text { No (\%) } \\
(n=16)\end{array}$ & p-value & $\begin{array}{c}\text { Yes (\%) } \\
(n=29)\end{array}$ & $\begin{array}{c}\text { No (\%) } \\
(n=104)\end{array}$ & p-value \\
\hline \multirow[t]{2}{*}{ Age(years) } & $<60$ & 53 & $46(86.8)$ & $7(12.1)$ & 0.734 & $11(20.8)$ & $42(79.2)$ & 0.811 \\
\hline & $\geq 60$ & 80 & $71(88.8)$ & $9(10.7)$ & & $18(22.5)$ & $62(77.5)$ & \\
\hline \multirow[t]{2}{*}{ Gender } & Male & 92 & $82(89.1)$ & 10(10.9) & 0.570 & $22(23.9)$ & $70(76.1)$ & 0.378 \\
\hline & Female & 41 & $35(85.4)$ & $6(14.6)$ & & $7(17.1)$ & $34(82.9)$ & \\
\hline \multirow[t]{2}{*}{ DM } & Positive & 18 & 16(88.9) & $2(11.1)$ & 1.000 & $5(27.8)$ & $13(72.2)$ & 0.543 \\
\hline & Negative & 115 & $101(87.8)$ & $14(12.2)$ & & $24(20.9)$ & $91(79.1)$ & \\
\hline \multirow[t]{2}{*}{$\operatorname{Size}(\mathrm{cm})$} & $<5$ & 65 & $54(83.1)$ & $11(16.9)$ & 0.090 & $10(15.4)$ & $55(84.6)$ & 0.080 \\
\hline & $\geq 5$ & 68 & 63(92.6) & $5(7.4)$ & & $19(27.9)$ & $49(72.1)$ & \\
\hline \multirow[t]{2}{*}{ Location } & Upper & 22 & 20(90.9) & $2(9.1)$ & 1.000 & $5(22.7)$ & $17(77.3)$ & 0.909 \\
\hline & Middle and lower & 111 & $97(87.4)$ & 14(12.6) & & $24(21.6)$ & $87(78.4)$ & \\
\hline \multirow[t]{2}{*}{ Histology } & Tubular carcinoma & 108 & $95(88.0)$ & $13(12.0)$ & 1.000 & $25(23.1)$ & $83(76.9)$ & 0.435 \\
\hline & Signet ring/mucinous & 25 & $22(88.0)$ & $3(12.0)$ & & $4(16.0)$ & $21(84.0)$ & \\
\hline \multirow[t]{2}{*}{ Curability } & Curative operation & 99 & $84(84.8)$ & $15(15.2)$ & 0.047 & $14(14.1)$ & $85(85.9)$ & $<0.001$ \\
\hline & Non-curative operation & 34 & $33(97.1)$ & $1(2.9)$ & & $15(44.1)$ & 19(55.9) & \\
\hline
\end{tabular}

PET, positron emission tomography, CT, computed tomography, FDG, fluorodeoxyglucose, LN, lymph nodes, DM, diabetes mellitus. 

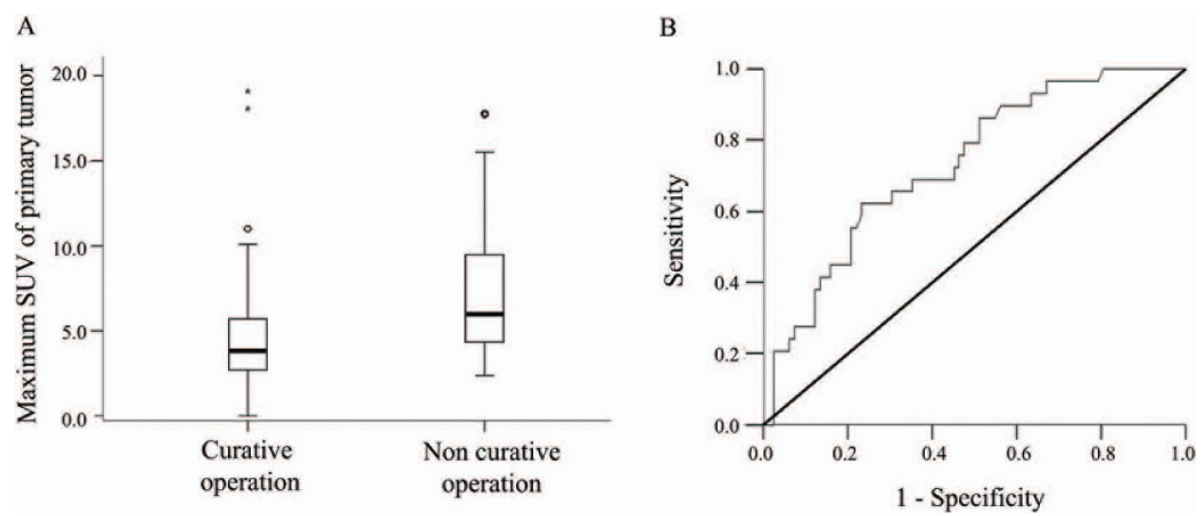

Figure 2 Maximum SUV of primary tumor related to curative or non-curative operation. A: Box plot of maximum SUV of primary tumor in patients with curative or non-curative surgery; the mean values were significantly different between the two groups in an independent $t$-test $(p<0.001)$. B: Receiver operator characteristics (ROC) curve of maximum SUV of primary tumor for predicting non-curative operation. The area under the curve was $0.730(p<0.001,0.629<95 \%$ C.I. $<0.831)$.

enhanced CT scan; 3) to evaluate other hidden simultaneous malignancies that are asymptomatic and undetectable by CT scanning. Contrary to above usage of PET-CT in gastric cancer, we focused on the prediction of surgical finding through the result of preoperative PET-CT. The results of our study suggested that treatment strategy of gastric cancer could be decided according to finding of FDG-PET CT.

With respect to preoperative PET/CT as a tool for surgical strategy decisions, the present study uncovered several relevant results. Using the semi-quantitative feature of FDG-PET/CT, the degree of FDG uptake of the primary tumor and the SUV was analyzed for prediction of curability. The mean SUV of the primary tumor in patients who underwent non-curative surgery was significantly higher than that of patients with curative surgery. Therefore, the SUV of the primary tumor might be a predictive factor for non-curative surgery; this is supported by the results of the ROC curve. When we defined a mean primary tumor SUV of greater than 5.0 and positive uptake of FDG in perigastric lymph nodes as cutoff values for prediction of non-curative resection, the sensitivity, specificity and accuracy were higher than those of enhanced CT scanning. Therefore, we find that FDG$\mathrm{PET} / \mathrm{CT}$ may be a tool for decisions concerning laparoscopic staging or neoadjuvant chemotherapy.

SUV values are common indices of tracer uptake in studies with PET, and can be calculated from the radioactivity of tumors following injection of fluorine ${ }^{18} \mathrm{~F}-\mathrm{FDG}$ according to body weight and physical decay [12]. The possibility of applying the SUV to preoperative PET/CT as a predictor for curability is explained by the following. The SUV may represent the growth rate of malignant tumors. Several reports have described that glucose utilization is higher in rapidly growing tumors than in less aggressive neoplasia $[13,14]$. In our study, the mean SUV was correlated with curability of advanced gastric cancer.

Diagnostic laparoscopy for the staging of gastric cancer has the benefit for diagnosis of radiographically occult metastatic disease. However, laparoscopic staging requires general anesthesia and many studies have reported that most patients who undergo laparoscopic staging also have to undergo laparotomy [15-17]. In addition, animal studies have shown that pneumoperitoneum due to laparoscopic examination could impair immunity and promote tumor growth [18-20]. Therefore, the routine use of laparoscopic

Table 3 Prediction of non-curative operation in patients who underwent operation $(n=133)$

\begin{tabular}{cccccc}
\hline & $\boldsymbol{n}$ & Sensitivity & Specificity & Accuracy & Positive predictive value \\
\hline $\begin{array}{c}\text { Enhanced CT Scan } \\
\quad \text { (Suspicious }\end{array}$ & 18 & 0.176 & 0.879 & 0.699 & 0.333 \\
non-curability) & & & & 0.426 \\
Tumor SUV > 5 & 54 & 0.676 & 0.687 & 0.684 & 0.500 \\
Tumor SUV > 7 & 24 & 0.353 & 0.879 & 0.744 & 0.530 \\
Tumor SUV > 9 & 17 & 0.265 & 0.919 & 0.752 & 0.517 \\
$\quad$ Local LN & 29 & 0.441 & 0.859 & 0.752 & 0.571 \\
SUV uptake (+) & & & 0.767 & \\
SUV > 5 and LN (+) & 21 & 0.352 & 0.910 & & \\
\hline
\end{tabular}

$\mathrm{CT}$, computed tomography, SUV, standardized uptake value, $\mathrm{LN}$, lymph nodes. 
staging for patients with advanced gastric cancer has been questioned. Several studies have recommended that laparoscopic staging be performed in patients with advanced primary tumors (overinvasion into muscle propria) and no significant metastatic lesion, and avoided if the tumor does not involve the gastroesophageal junction and lymph node metastasis is absent on spiral CT or endoscopy ultrasound (EUS) [6,21]. However, the results of CT or EUS are frequently subjective depending on the radiologist or endoscopist, whereas PET/CT can establish objective information such as the uptake of FDG in primary tumors or lymph nodes and the degree of uptake presented as the SUV.

In terms of FDG uptake in local lymph nodes, although PET/CT added anatomical information of lymph node enlargement, PET scanning is limited in its ability to separate a local lymph node from a primary tumor due to intense tracer accumulation and ill-defined anatomical boundaries [22]. Metastatic local lymph nodes were identified by PET/CT when there were enlarged lymph node lesions with FDG uptake occurring separately from primary tumors. In addition, there enlarged or conglomerated lymph nodes can lead to unresectablilty due to the invasion of the metastatic nodes into the pancreas and major vessels like hepatic artery or celiac trunk. Therefore, although the positive rate of metastatic lymph nodes in $\mathrm{PET} / \mathrm{CT}$ is not high, it may indicate as aggressive as these gastric cancers are difficult to cure with resection. In our study, positive lymph node metastasis in PET/CT was related to non-curative surgery; this might have higher predictive accuracy for non-curative surgery that the SUV of the primary tumor alone.

Our study has several limitations. First, the number of enrolled patients might be too small to confirm the clinical validity of PET/CT for gastric cancer. Therefore, studies enrolling larger populations should be planned in order to confirm the correlation between preoperative PET/CT and operative findings. Second, the criteria for non-curative surgery might be subjective. In this study, gastric cancer with definite distant metastatic lesions (M1) or with surgical findings of invasion into the pancreatic head were necessarily defined as non-curative surgery. Pancreaticoduodenectomy as a curative surgery for pancreatic invasions of gastric cancer requiring are controversial due to high operative morbidity and mortality [23,24]. Moreover, no results from clinical trials have confirmed the benefit of pancreaticoduodenectomy for gastric cancer. Third, although previous studies have reported a difference in FDG uptake rate according to the histological type of gastric cancer $[9,25]$, this was not observed in our study. We believe that confining our enrollment of patients to those with advanced gastric cancer might mask the difference in FDG uptake by histological type, since the tumor size and depth of invasion can effect on the FDG uptake [9].

\section{Conclusions}

Despite these limitations, our results show that high FDG uptake rate of the primary tumor and local lymph nodes is related to non-curable surgery. High SUV of the primary tumor and positive FDG uptake of local lymph nodes in PET/CT could predict non-curable surgery in locally advanced gastric cancer with higher specificity, accuracy and positive predictive values than those achieved by CT scan. Therefore, we suggest that gastric cancer patients showing high SUV in the primary tumor and positive FDG uptake in local lymph nodes at PET/ CT should be subjected to neoadjuvant chemotherapy or laparoscopic staging in order to avoid unnecessary laparotomy. Furthermore, we will evaluate the correlation between preoperative PET/CT and post-operative prognosis through follow-up of the enrolled patients to enhance the clinical benefit of PET/CT.

\section{Author details}

${ }^{1}$ Department of Surgery, Ajou University, School of Medicine, Suwon, Korea. 2Department of Nuclear Medicine, The Catholic University of Korea, College of Medicine, Seoul, Korea. ${ }^{3}$ Department of Surgery, The Catholic University of Korea, College of Medicine, Seoul, Korea.

\section{Authors' contributions}

$\mathrm{HH}$ : Analysis of the data and drafting of the manuscript. SHK: Interpretation of data. WK, KYS, CHP: Revise it critically for important intellectual content. HMJ: Concept and design of the manuscript. All authors read and approved the final manuscript.

\section{Competing interests}

The authors declare that they have no competing interests.

Received: 26 May 2010 Accepted: 11 October 2010

Published: 11 October 2010

\section{References}

1. Kim JP: Current status of surgical treatment of gastric cancer. J Surg Oncol 2002, 79:79-80

2. Hartgrink $\mathrm{HH}$, Bonenkamp HJ, van de Velde $\mathrm{CJ}$ : Influence of surgery on outcomes in gastric cancer. Surg Oncol Clin N Am 2000, 9:97-117.

3. Iriyama K, Nishiwaki $H$, Mori $H$, Suzuki H: Prediction of post-operative survival time by multivariate analysis in patients with advanced cancer of the stomach. Int Surg 1986, 71:73-75.

4. Chen J, Cheong JH, Yun MJ, Kim J, Lim JS, Hyung WJ, Noh SH: Improvement in preoperative staging of gastric adenocarcinoma with positron emission tomography. Cancer 2005, 103:2383-2390.

5. Ajani J, Bekaii-Saab T, D'Amico TA, Fuchs C, Gibson MK, Goldberg M, Hayman JA, Ilson DH, Javle M, Kelley S, Kurtz RC, Locker GY, Meropol NJ Minsky BD, Orringer MB, Osarogiagbon RU, Posey JA, Roth J, Sasson AR, Swisher SG, Wood DE, Yen Y: Gastric Cancer Clinical Practice Guidelines. J Natl Compr Canc Netw 2006, 4:350-366.

6. Burke EC, Karpeh MS, Conlon KC, Brennan MF: Laparoscopy in the management of gastric adenocarcinoma. Ann Surg 1997, 225:262-267.

7. Kinkel K, Lu Y, Both M, Warren RS, Thoeni RF: Detection of hepatic metastases from cancers of the gastrointestinal tract by using noninvasive imaging methods (US, CT, MR imaging, PET): a metaanalysis. Radiology 2002, 224:748-756.

8. Yeung HW, Macapinlac H, Karpeh M, Finn RD, Larson SM: Accuracy of FDGPET in Gastric Cancer. Preliminary Experience. Clin Positron Imaging 1998, 1:213-221.

9. Mochiki E, Kuwano $H$, Katoh $H$, Asao $T$, Oriuchi $N$, Endo K: Evaluation of 18F-2-deoxy-2-fluoro-D-glucose positron emission tomography for gastric cancer. World J Surg 2004, 28:247-253. 
10. Dassen AE, Lips DJ, Hoekstra CJ, Pruijt JF, Bosscha K: FDG-PET has no definite role in preoperative imaging in gastric cancer. Eur J Surg Oncol 2009, 35:449-455.

11. Kim EY, Lee WJ, Choi D, Lee SJ, Choi JY, Kim BT, Kim HS: AThe value of $\mathrm{PET} / \mathrm{CT}$ for preoperative staging of advanced gastric cancer: Comparison with contrast-enhanced CT. Eur J Radiol 2010.

12. Woodard HQ, Bigler RE, Freed B: Letter: Expression of tissue isotope distribution. J Nucl Med 1975, 16:958-959.

13. Adler LP, Blair HF, Williams RP, Pathria MN, Makley JT, Joyce MJ, al-Kaisi N, Miraldi F: Grading liposarcomas with PET using [18F]FDG. J Comput Assist Tomogr 1990, 14:960-962.

14. Duhaylongsod FG, Lowe VJ, Patz EF, Vaughn AL, Coleman RE, Wolfe WG: Lung tumor growth correlates with glucose metabolism measured by fluoride-18 fluorodeoxyglucose positron emission tomography. Ann Thorac Surg 1995, 60:1348-1352.

15. Bemelman WA, van Delden OM, van Lanschot JJ, de Wit LT, Smits NJ, Fockens P, Gouma DJ, Obertop H: Laparoscopy and laparoscopic ultrasonography in staging of carcinoma of the esophagus and gastric cardia. J Am Coll Surg 1995, 181:421-425.

16. Hulscher JB, Nieveen van Dijkum EJ, de Wit LT, van Delden OM, van Lanschot JJ, Obertop H, Gouma DJ: Laparoscopy and laparoscopic ultrasonography in staging carcinoma of the gastric cardia. Eur J Surg 2000, 166:862-865.

17. Romijn MG, van Overhagen H, Spillenaar Bilgen EJ, ljzermans JN, Tilanus HW, Lam®ris JS: Laparoscopy and laparoscopic ultrasonography in staging of oesophageal and cardial carcinoma. Br J Surg 1998, 85:1010-1012.

18. Bouvy ND, Marquet RL, Jeekel J, Bonjer HJ: Laparoscopic surgery is associated with less tumour growth stimulation than conventional surgery: an experimental study. BrJ Surg 1997, 84:358-361.

19. Gitzelmann CA, Mendoza-Sagaon M, Talamini MA, Ahmad SA, Pegoli W, Paidas CN: Cell-mediated immune response is better preserved by laparoscopy than laparotomy. Surgery 2000, 127:65-71.

20. Iwanaka T, Arkovitz MS, Arya G, Ziegler MM: Evaluation of operative stress and peritoneal macrophage function in minimally invasive operations. J Am Coll Surg 1997, 184:357-363.

21. Sarela Al, Lefkowitz R, Brennan MF, Karpeh MS: Selection of patients with gastric adenocarcinoma for laparoscopic staging. Am J Surg 2006, 191:134-138.

22. Lerut T, Flamen P: Role of FDG-PET scan in staging of cancer of the esophagus and gastroesophageal junction. Minerva Chir 2002, 57:837-845.

23. Buchholtz TW, Welch CE, Malt RA: Clinical correlates of resectability and survival in gastric carcinoma. Ann Surg 1978, 188:711-715.

24. Saka M, Mudan SS, Katai H, Sano T, Sasako M, Maruyama K: Pancreaticoduodenectomy for advanced gastric cancer. Gastric Cancer 2005, 8:1-5.

25. Yoshioka T, Yamaguchi K, Kubota K, Saginoya T, Yamazaki T, Ido T, Yamaura G, Takahashi H, Fukuda H, Kanamaru R: Evaluation of 18F-FDG PET in patients with advanced, metastatic, or recurrent gastric cancer. $J$ Nucl Med 2003, 44:690-699.

doi:10.1186/1477-7819-8-86

Cite this article as: Hur et al:: The efficacy of preoperative PET/CT for prediction of curability in surgery for locally advanced gastric carcinoma. World Journal of Surgical Oncology 2010 8:86.

\section{Submit your next manuscript to BioMed Central and take full advantage of:}

- Convenient online submission

- Thorough peer review

- No space constraints or color figure charges

- Immediate publication on acceptance

- Inclusion in PubMed, CAS, Scopus and Google Scholar

- Research which is freely available for redistribution

Submit your manuscript at www.biomedcentral.com/submit
Biomed Central 